\section{Cahiers de Narratologie}

Analyse et théorie narratives

33 | 2018

L'Art du roman chez Umberto Eco

\title{
Raconter ou prouver. Récits de découvertes et de non-découvertes de grottes ornées
}

\section{Sylvie Grenet and Noël Coye}

\section{(2) OpenEdition}

\section{Journals}

Electronic version

URL: http://journals.openedition.org/narratologie/8425

DOI: $10.4000 /$ narratologie. 8425

ISSN: 1765-307X

Publisher

LIRCES

\section{Electronic reference}

Sylvie Grenet and Noël Coye, "Raconter ou prouver. Récits de découvertes et de non-découvertes de grottes ornées », Cahiers de Narratologie [Online], 33 | 2018, Online since 23 July 2018, connection on 10 December 2020. URL : http://journals.openedition.org/narratologie/8425 ; DOI : https://doi.org/ $10.4000 /$ narratologie.8425

This text was automatically generated on 10 December 2020.

Article L.111-1 du Code de la propriété intellectuelle. 


\title{
Raconter ou prouver. Récits de découvertes et de non-découvertes de grottes ornées
}

\author{
Sylvie Grenet and Noël Coye
}

«[L'histoire] est écrite en vue de raconter, non de prouver»

«scribitur ad narrandum, non ad probandum [...]»

(Quintilien, Institution oratoire, X, 1, 31, trad.

Collection des Universités de France)

Dans le dernier tiers du XIX ${ }^{e}$ siècle, la discipline préhistorique se construit et adopte le régime de scientificité, fondé sur les principes de validité, d'objectivité et de représentativité (Boudreau et Arseneault 1994 ; Lecompte et Goetz 1982 ; Drapeau 2004). Les découvertes sont interrogées au moyen de protocoles qui permettent de les authentifier, et de les replacer dans une connaissance globale, cohérente et structurée, élaborée à partir des cadres de savoir issus des sciences de la nature et des sciences de l'homme (Hurel 2007, Coye 1997, Richard 2008). Le protocole d'étude conduit à faire accéder la découverte, qui appartient au domaine du tangible et de l'observable, au régime de scientificité, en l'intégrant dans une configuration du savoir.

Dans ce processus, la découverte peut revêtir deux natures différentes: soit être intégrable immédiatement dans cette configuration (qui est elle-même déterminante dans le signalement de la découverte), soit être un élément perturbateur ne pouvant y trouver place, voire la remettant fondamentalement en cause. Cette dichotomie entre découverte, au sens de réalité observable, et connaissance au sens de réalité systématisée, renvoie par ailleurs à une pluralité d'acteurs - découvreurs, scientifiques, médiateurs qui utilisent des processus narratifs propres à la catégorie à laquelle ils appartiennent pour rendre compte de ces découvertes.

3 Cette pluralité du statut de la découverte, associée à la multiplicité des acteurs, instaure un régime de tension particulier entre les modalités du récit et la nécessité de la preuve. A soixante ans d'intervalle, alors même que la nature des preuves de scientificité a 
évolué, les récits de découverte d'Altamira et de Lascaux constituent des moments clés ayant fixé cette tension. Plus récemment, elle s'est prolongée dans ce que nous appellerons la non-découverte d'un «nouveau Lascaux » à Montignac en Dordogne, entre la multiplication des formes du récit et l'impossibilité de la validation scientifique.

\section{Altamira : la légende comme produit du régime de scientificité}

4 A l'époque de la découverte d'Altamira (Cantabrie, Espagne), le régime de scientificité en préhistoire repose sur une élaboration interne à la communauté savante. Les publications - revues et actes de congrès internationaux - sont des lieux de confrontation où production et diffusion de l'information cohabitent dans une même sphère (Coye 2000). L'insertion d'Altamira dans le régime de scientificité est longue, complexe, et marquée par la polémique. D'abord, la découverte s'effectue en deux temps. Les fouilles archéologiques y débutent en 1875 mais les peintures du plafond de la grande salle sont découvertes en 1879. A ces deux dates correspondent deux temporalités et deux types d'impact différents. En effet, si le mobilier est immédiatement identifié sans problèmes majeurs, le débat sur l'authentification des peintures ouvre un chantier polémique, puisque ce sont les peintures d'Altamira qui vont lancer un débat qui durera près de 25 ans sur l'authenticité de l'art pariétal (Richard 1993, Coye 1997, 241-248)1.

La prégnance du modèle de validation scientifique par le biais de la littérature savante aboutit à l'élaboration d'un récit de scientificité particulier, dont les éléments constitutifs sont établis par Marcelino Sanz de Sautuola (1831-1888), découvreur et premier explorateur de la grotte, dans sa publication de 1880 : Breves apuntes sobre algunos objetos prehistóricos de la provincia de Santander. Cette publication suit de très peu la découverte et la phase d'observation. Dans un contexte où l'authenticité des figures pose rapidement question, Sautuola ne présente pas un récit de découverte, mais un ensemble structuré d'observations, qui reste au plus près de la description brute des objets, des figures et des circonstances qui l'ont amené à voir les peintures : «Je n'occulte pas de mon esprit, que certains de mes lecteurs puissent avoir des doutes sur ces dessins et peintures dont je me suis occupé [...] Tout est possible, mais si l'on prend la chose au sérieux, il apparait que cette opinion n'est pas admissible. Cette grotte était totalement méconnue jusque qu'à ce que j'y entre pour la première fois, en étant l'un des premiers visiteurs. (...) Les peintures [...] de la première galerie, je ne les découvris que l'année dernière en 1879, car à la vérité je n'avais pas examiné la voûte avec autant d'attention, et parce que pour les voir il faut rechercher les angles de vue, surtout s'il y a peu de lumière. [...] Il paraît inadmissible que quelqu'un par distraction soit allé là pour peindre des figures indéchiffrables, et pour celles de la première galerie, il est impensable de supposer qu'à une date récente quelqu'un ait eu le caprice de s'enfermer dans ce lieu pour reproduire des peintures d'animaux inconnus dans ce pays à notre époque. » (Sautuola 1880, 22, notre trad.)

La «modestie formelle et insistante» (Lasheras et las Heras 2004, 119) de l'auteur ne l'empêche pas de proposer une intégration de la grotte dans la connaissance préhistorienne de son époque, en employant une méthodologie déductive, fondée sur la typologie des objets présents dans le sol, et sur une comparaison avec les pièces d'art mobilier déjà connues par ailleurs : « De tout ce qui précède nous pouvons déduire, avec fondement que [la grotte d'Altamira appartient], sans aucun doute, à l'époque désignée 
avec le nom de paléolithique, c'est-à-dire celle de la pierre taillée, autrement dit, la primitive qui peut se référer à ces montagnes.» (Sautuola 1880, 23, notre trad.). Cependant, il laisse aux hommes de science le pouvoir de l'insérer définitivement dans le régime de scientificité : «Il reste donc, pour d'autres personnes plus instruites, à faire l'étude consciencieuse des données que j'ai mentionnées à la légère ; pour l'auteur de ces lignes la satisfaction d'avoir recueilli une grande partie d'objets si curieux pour l'histoire de ce pays [...] étant suffisante, provoquant ainsi un motif pour que les hommes de science fixent leur attention sur cette province, digne d'être étudiée plus qu'elle ne l'a été jusqu'à ce jour. » (Sautuola 1880, 24, notre trad.).

7 A partir de cette première publication, caractérisée par l'absence du récit de découverte, et la position ambiguë du découvreur qui, tout en développant un discours scientifique, s'en remet au verdict du monde savant auquel il déclare ne pas appartenir, le débat va se développer dans l'institution scientifique par la construction conjointe d'un récit de découverte et d'une démonstration scientifique, qui vont agir en interaction.

En réponse à la publication de Sautuola, Edouard Harlé (1850-1922) publie en 1881 «La grotte d'Altamira près de Santander " (Harlé 1881). Il déploie une analyse scientifique, fondée sur des arguments techniques et stylistiques, pour démontrer que les peintures du grand plafond sont «fort récentes » et « qu'elles ont été faites dans l'intervalle des deux premières visites de M. de Sautuola, de 1875 à 1879 » (Harlé 1881, 282). Ce n'est pas Sautuola qui répond à cette démonstration, mais un savant institutionnel, Juan Vilanova y Piéra (1821-1893), professeur de géologie et de paléontologie à l'université de Madrid, qui s'exprime devant le Congrès de l'association française pour l'avancement des sciences (AFAS) réuni en 1882 à la Rochelle. Fervent partisan de l'authenticité de la grotte, celui-ci répond point par point aux arguments de Harlé et oppose à l'accusation stigmatisant la découverte tardive des peintures un élément d'un autre ordre, introduisant le récit de découverte dans le débat : «M. de Sautuola déclare avec loyauté, n'avoir pas bien regardé le plafond de la première galerie et les dessins de la deuxième dans ses premières visites à la caverne, préoccupé comme il l'était par les richesses archéologiques qu'il trouvait dans le dépôt (...) ; et il a dû bien plus tard être averti par sa jeune fille pour reconnaître l'existence de ces objets d'art anciens » (Vilanova y Piera 1883, 671).

9 Le recours à la figure de la jeune enfant de Sautuola a d'emblée pour les contemporains une résonance qui va au-delà de la simple mention anecdotique. La figure de l'enfant est en effet déjà très évocatrice à la fin du XIX $\mathrm{XI}^{\mathrm{e}}$ siècle, tant elle « s'identifie à l'expérience du bonheur élémentaire, à l'extase des premières sensations aigües, à l'émerveillement devant le monde encore inconnu. L'enfance est la période de vie authentique, où tous les mouvements sont spontanés et viennent du cœur» (Bethlenfalvay 1979, 24). En transférant la découverte de l'adulte vers l'enfant, et en jouant sur l'innocence et l'authenticité d'un regard qui peut seul découvrir des objets totalement nouveaux, cet argument tente probablement, par des moyens détournés, de déjouer la suspicion de fraude. A cette étape de la polémique, le récit de la découverte a pourtant du mal à se connecter avec le débat scientifique. Les observations conduites à Altamira sont marginalisées, voire ostracisées, comme c'est le cas lors du Congrès international d'anthropologie et d'archéologie préhistoriques de Lisbonne en 1884. La longue communication présentée par Vilanova ne se retrouve dans les Actes que sous la forme d'une brève mention : «M. Vilanova invite les membres du Congrès à visiter avec lui les cavernes de Santillana, près Santander, dont les parois et le plafond offrent des gravures 
coloriées où l'on reconnaît l'aurochs. Cette découverte est due à M. Marcelino de Santuola (sic). » (Anonyme 1884).

10 Au tournant des XIXe et $\mathrm{XX}^{\mathrm{e}}$ siècles, deux vagues de découvertes françaises apportent des éléments qui concourent à intégrer la compréhension de l'art pariétal dans la connaissance globale élaborée par les préhistoriens: au milieu des années 1890, les découvertes de la Mouthe (Dordogne, 1895), Pair-non-Pair (Gironde, 1896) et Marsoulas (Haute-Garonne, 1897), en 1901 les découvertes de Font-de-Gaume et des Combarelles en Dordogne. L'ensemble de ces éléments aboutit à la reconnaissance de l'art pariétal au cours du Congrès AFAS de Montauban en 1902. Ce mouvement rétrospectif fait entrer Altamira dans le concert de la préhistoire.

11 C'est, là encore, dans l'arène des publications savantes que se déroule le débat sur l'authenticité de la grotte. Il ne prend cependant plus la forme d'un récit de découverte, mais d'un mea culpa, introduisant ainsi dans l'espace de la validation scientifique l'affirmation de la possibilité d'une erreur de jugement personnelle, soulignant en creux, par la même occasion, le prestige d'un savant qui commence à acquérir un pouvoir de légitimation personnel. Une des premières reconnaissances de la grotte est en effet due à Emile Cartailhac (1845-1921)², détracteur de la première heure aux côtés d'Edouard Harlé. Dans un texte demeuré célèbre et publié en 1902, Les cavernes ornées de dessins. La grotte d'Altamira, Espagne. "Mea culpa» d'un sceptique, Emile Cartailhac reconnaît l'authenticité de la grotte. Préludant au Congrès de Montauban et à la mission de relevés que Cartailhac effectuera sur place avec l'abbé Breuil quelques mois plus tard, le Mea culpa entérine le changement de conviction personnelle de l'auteur. Celui-ci se fonde sur une démarche intellectuelle qui soumet les découvertes successives intervenues depuis 1895 au régime de validation qui est celui de l'archéologie préhistorique de son époque : il ne se rend pas sur place pour entériner l'authenticité des peintures, mais se fonde sur les observations qu'il a effectuées en France et sur la typologie des grottes ornées françaises déjà établie, pour authentifier Altamira. Les congressistes de l'AFAS suivent la même méthode pour intégrer l'art pariétal au régime de scientificité. La démonstration est parachevée, comme l'administration de la preuve l'exige alors, par une reconnaissance in situ, via la mise en place d'« excursions » dans les trois grottes ornées de Dordogne (La Mouthe, Font-deGaume et Les Combarelles). La différence réside cependant dans le fait que Cartailhac revendique la reconnaissance comme un acte personnel, écrivant: « je dois, pour ce qui me concerne, faire amende honorable à M. de Sautuola 》(Cartailhac 1902, 354), alors que le congrès de l'AFAS marque l'adhésion collective de la communauté des préhistoriens. Faisant le compte-rendu des excursions, dans les Actes du colloque, Emile Rivière conclut: "Bref, nous croyons pouvoir dire, sans être démentis par aucun d'eux, que l'authenticité paléolithique de tous les dessins gravés et peints des trois grottes (...) ne laissent désormais aucun doute dans l'esprit de nos collègues» (Rivière 1902, 272).

La résolution du débat scientifique conduit dans les publications savantes est ainsi sanctionnée par l'examen in situ. Cependant, les autres types de récit esquissés pour justifier de l'authenticité des figures d'Altamira, perdurent, et prennent une importance grandissante alors même que le débat scientifique est clos. Le personnage de l'enfant, passé inaperçu pendant toute la durée du débat sur l'authenticité, ne cesse de prendre davantage d'importance une fois le débat conclu. Le véritable rôle joué par Maria, la fille de Sautuola, demeure difficile à établir avec précision. Les écrits de l'abbé Breuil, un des principaux acteurs de la promotion scientifique d'Altamira, livrent des mentions ambiguës. La publication que celui-ci consacre à la grotte avec Emile Cartailhac en 1906 
reprend à plusieurs reprises, sans les développer, les éléments auparavant présentés par Vilanova $^{3}$. En revanche, dans son Autobiographie, rédigée au début des années 1950 et demeurée inédite, l'abbé Breuil raconte sa rencontre en 1902 avec Maria, devenue Mme Botín y Lopez : « Mme Botin (sic), qui avait, la première, à 7 ans, accompagnant son père, appelé son attention sur les fresques du grand plafond, n'avait gardé aucun souvenir de l'événement $»^{4}$.

Quoi qu'il en soit de la réalité de l'acte de Maria, les récits ne cessent de développer son rôle dans la découverte, avec de nombreux détails contradictoires, plus particulièrement quant à son âge au moment de la découverte (Gaussel 1997). De même que la " conversion » individuelle d'Emile Cartailhac, incarnée par son Mea culpa, a été bien souvent considérée comme l'élément déterminant, occultant la conversion collective marquée par le congrès de l'AFAS, la figure de Maria acquiert un poids tel qu'elle devient non seulement un élément central du récit de la découverte, mais également du processus de reconnaissance de l'art pariétal. Pour citer un exemple parmi d'autres, dans son article "Le poète dans la caverne ", l'anthropologue Daniel Fabre va jusqu'à nouer ces deux éléments et à attribuer à la rencontre avec Maria la conversion de Cartailhac qui, nous l'avons vu, est en fait bien antérieure (Fabre 2008, 141, note 15).

14 A travers le recours à l'enfant, Vilanova a mis en place un mode d'authentification de l'œuvre, qui a des résonances encore de nos jours. On a pu l'interpréter de différentes manières au XX $\mathrm{X}^{\mathrm{e}}$ siècle, comme l'apparition du thème de l'innocence de l'art des premiers hommes, qui serait reconnaissable uniquement par un autre œil innocent (Fabre 2008, Fraenkel 2010). On peut aussi y voir, dans le contexte des avant-gardes artistiques de la fin du XIXe siècle et du début du XX ${ }^{e}$ siècle qui revendiquent l'enfance comme source d'inspiration (Pernoud 2003), un signe de l'affranchissement des normes, et partant, une possible critique du poids du régime de scientificité, qui empêcherait, par excès de méthodologie et de références, de reconnaître l'authenticité de la grotte.

15 La figure de l'enfant en est même arrivée à devenir la figure héroïque et légendaire analysée par Gérard Bras, élément d'équilibre qui permet d'ajuster l'ensemble des parties (Bras 2006) $)^{5}$. L'un d'entre nous a été membre du Collège d'experts de Lascaux Centre International de l'Art Pariétal, abritant le fac-simile intégral de la grotte et inauguré en décembre 2016 à Montignac. Un des dispositifs de médiation intitulé « Théâtre de l'art pariétal » y retrace notamment le débat sur la reconnaissance de l'art pariétal. Dans ce contexte, représenter la scène de la découverte d'Altamira supposait de statuer sur le rôle exact de Maria. Les sources historiques, composées de récits ambigus et polymorphes, ne permettaient pas d'établir celui-ci avec certitude. Une option substituant la construction de l'observation scientifique au récit anecdotique a donc été examinée. Celle-ci n'a pas recueilli l'adhésion de l'équipe des scénographes qui arguaient de l'intérêt de la dimension dramatique $\mathrm{du}$ récit traditionnel. Face à l'impossibilité d'invalider historiquement le récit légendaire, il a été décidé collectivement de conserver celui-ci et de l'intégrer au dispositif de médiation.

16 Au compte-rendu d'un regard érudit qui ne cesse d'errer d'un espace à un autre jusqu'à, un jour, parvenir à la révélation en s'orientant vers la voûte ornée, s'oppose le récit d'un regard innocent, pur et authentique, qui découvre et identifie d'emblée les figures. Né de manière presque anecdotique de la nécessité de produire un nouveau récit d'observation pour pallier l'échec d'un premier récit, le récit de la découverte par la jeune fille ne cesse d'occuper l'espace, au point de devenir aujourd'hui incontournable pour tous les acteurs. 
Avec Altamira, le régime de scientificité a fait naître une légende, qui vit désormais de sa vie propre, tout en dialoguant avec lui.

\section{L'insertion de la légende dans le régime de scientificité : la naissance du mythe de Lascaux}

17 La découverte de Lascaux surgit dans une nouvelle configuration du régime de scientificité appliqué à l'art pariétal. Dans les années 1940, les publications savantes ne sont plus le lieu exclusif des débats, et leur caractère collégial est dominé par la parole de celui qui est maintenant le "pape de la préhistoire ", l'abbé Henri Breuil. Erigées en argument d'autorité, la présence et la parole de celui-ci suffisent désormais pour authentifier une grotte ornée, dans un processus intégratif où autorité fait preuve. En outre, les découvertes archéologiques spectaculaires sont entrées depuis les années 1920, en particulier avec la découverte de la tombe de Toutankhamon, dans le domaine de l'événementiel, et font désormais partie intégrante du discours journalistique.

18 La temporalité de Lascaux tranche radicalement avec celle d'Altamira. Ici, l'immédiateté l'emporte. La grotte est découverte par de jeunes garçons le 12 septembre $1940^{6}$, dans les environs du village de Montignac en Dordogne, dans un milieu baigné par la préhistoire. Les découvreurs identifient tout de suite la nature de ce qu'ils ont sous les yeux, et une première pré-validation intervient le 16 septembre, par l'intermédiaire de leur instituteur, Léon Laval. Ce dernier prévient aussitôt l'abbé Breuil, qui arrive sur les lieux cinq jours plus tard, le 21, et authentifie immédiatement les peintures. La grotte entre ainsi directement dans le régime de scientificité, sans qu'il y ait de polémique sur son authenticité.

19 A Lascaux, les récits d'authentification et d'analyse de l'art pariétal relèvent directement du régime de scientificité ; c'est avec eux que les récits de médiation - et notamment les premiers récits journalistiques de la découverte - vont devoir composer. Nous avons choisi ici de privilégier une étude de la construction médiatique, et du temps très court de restitution, parce que celui-ci permet d'étudier la manière dont le récit de découverte a pu être élaboré, quasiment à chaud, par des médiateurs chargés de rapporter l'événement. Les récits produits plus tardivement par deux des découvreurs, une lettre rédigée par Marcel Ravidat (1922-1995) en 1983 et publiée en 1990 dans une bande dessinée, Le secret des bois de Lascaux (Félix et Bigotto 1990), et une conférence donnée par Jacques Marsal (1926-1989) au musée de l'Homme en 1986, fournissent un contrepoint aussi bien temporel que typologique et thématique.

Brigitte et Gilles Delluc ont accompli un précieux travail de récolement et de signalement de la presse contemporaine, publié dans Le Bulletin de la Société Historique et Archéologique du Périgord en 2012 (Delluc B. et Delluc G. 2012, 551-577). Ils dénombrent neuf articles parus dans les quinze jours qui suivent la découverte : deux articles coup sur coup dans Le Petit Parisien, datés du 25 puis du 26 septembre, un dans Paris-Soir et un autre dans Le Journal le 27 septembre, un deuxième dans Le Journal le 28 septembre, deux autres articles dans L'Echo du Centre, les 27 et 28 septembre, un dans Le Petit Gaillard (journal de Brive) le 28 et un dernier dans L'Argus du Périgord (journal de Périgueux), daté du 2 octobre. Cet inventaire peut être complété par un autre article, paru dans La Petite Gironde, le 29 septembre. 
21 Le premier article du Petit Parisien du 25 septembre 1940, signé André Le Bret, fixe une modalité de récit qu'on retrouve ensuite dans tous les autres. Il montre une juxtaposition entre une narration de type événementiel et une narration reprenant la structure diégétique du conte. La narration événementielle série les différentes étapes de la découverte: promenade des jeunes gens, intervention d'un chien qui les guide vers un trou, descente dans la grotte, vision des figures: "C'était le 12 septembre. Quatre gars d'ici, Ravidat, Marsal, Amiel [au lieu de Agniel], Coincas [au lieu de Coencas], suivaient un renard non loin du château de Lascaux qui appartient à Mme de la Rochefoucauld. Soudain, leur chien s'arrête devant un trou. L'un d'eux jette alors une pierre dont la chute, à leur surprise, se répercute en échos... Tous se regardent et joyeux, s'exclament : une grotte ! parions que c'est une grotte.».

Mais le merveilleux sourd du récit événementiel. Les épisodes de la découverte semblent coïncider naturellement avec la structure diégétique d'un conte, telle qu'elle pu être analysée par Paul Larivaille dans son schéma quinaire (Larivaille 1974). Paul Larivaille analyse la structure des contes selon cinq étapes : un état initial fait d'équilibre (1) est bouleversé par un détonateur (2), qui provoque une action (3). Cette action entraîne des conséquences (4) qui vont aboutir à un état final de nouvel équilibre (5). Si on lit le récit de la découverte de Lascaux selon ce schéma, on peut considérer que la découverte provoque un changement de l'état initial des quatre jeunes gens, qui entraîne pour eux comme pour le monde un bouleversement qui va engendrer des conséquences irrémédiables dans le domaine de la connaissance et dans les destins particuliers. Interviennent même des éléments qui sont du ressort du conte merveilleux : dans une forêt, les quatre découvreurs étaient initialement partis à la recherche d'un trésor ${ }^{7}$ et un animal les guide dans un monde peuplé de "fresques fantasmagoriques ${ }^{8}$ dont ils ressortent transformés, puisque de découvreurs, ils deviennent rapidement les gardiens $\mathrm{du}$ lieu. Le récit de la découverte entre dans le domaine du conte initiatique et merveilleux, par le récit journalistique, qui n'est pas uniquement événementiel, mais coïncide avec une structuration narrative initiatique prégnante dans les esprits, devenue réalité.

La juxtaposition entre le conte et la réalité connaît un autre développement dans le courtmétrage réalisé en 1942 par la Gaumont, et intitulé « La Nuit des temps »" Se rattachant au genre du docufiction, celui-ci renforce le caractère initiatique du récit de découverte par l'ajout d'éléments fictifs. L'action se divise en deux parties : la première est consacrée au récit de la découverte, et l'autre, à la description et à l'interprétation des figures peintes. Si les éléments d'un récit d'initiation apparaissent bien dans le film, ils entretiennent un rapport particulier à la réalité des faits qui ont été vécus par les protagonistes eux-mêmes. Les découvreurs sont interprétés par des acteurs, Léon Laval étant le seul à incarner son propre personnage. Le scénario développe de plus une narration s'écartant notablement $d u$ récit originel, notamment en ce qui concerne l'événement déclencheur, qui est très proche des récits initiatiques. En effet, ceux-ci débutent souvent par une transgression à l'ordre établi : le héros se révolte, refuse une condition qui lui est imposée, et part à l'aventure. Dans une des premières scènes du film, Léon Laval punit un de ses élèves pour ne pas s'être intéressé à ses cours sur la préhistoire. C'est lorsque l'enfant, le plus hermétique à la préhistoire, se dérobe à son pensum, copier 100 fois la phrase «je ne connais rien à l'histoire de l'homme des cavernes ", et part gambader dans la colline, qu'il est le premier à entrer dans la grotte et à découvrir les figures de la salle des taureaux. Cet élément amplifie ainsi le caractère 
transgressif à l'origine de la découverte, rapprochant encore plus le récit de la structure du conte.

Ce film illustre les multiples fonctions que les protagonistes ont revêtues dès les premiers jours, dans une ambiance enfiévrée propice au renversement des rôles, telle que l'a rapportée Léon Laval : « Nos jeunes gens couchaient tous les soirs sous la tente au seuil du trésor à sauvegarder. L'abbé Breuil vivait avec nous : la radio, les journalistes venaient nous interviewer et les habitants de Montignac et des environs accouraient en foule, malgré les difficultés de la descente. L'abbé Breuil faisait de véritables conférences en plein air au public pèlerin qui l'écoutait avec recueillement et comprenait l'importance de la trouvaille $»^{10}$.

Dans cette atmosphère, la figure savante de l'instituteur, devenu acteur d'un docu-fiction, n'est pas été la seule à subir des transformations. La figure du préhistorien se plie également à ces métamorphoses. Dans le régime de scientificité désormais classique, tel que Sautuola l'avait intégré, les découvreurs ne sont pas ceux qui travaillent sur ou dans la grotte, les scientifiques prenant le relai pour valider la découverte et tempérer les ardeurs de l'imagination du public, en veillant à replacer fidèlement les figures dans leur contexte. Or, à Lascaux, l'abbé Breuil, s'il donne sa caution scientifique, endosse un rôle loin d'être modérateur, en dotant le lieu d'une survalorisation à la fois esthétique et sacrée par sa qualification de "Chapelle Sixtine de la préhistoire $»^{11}$. De même, le rôle des jeunes garçons changera au fil du temps. On l'a vu, dans un premier temps, de découvreurs, ils deviennent d'abord les gardiens du lieu, en plantant une tente pour éviter que les curieux n'affluent en désordre. Puis deux d'entre eux, Jacques Marsal et Marcel Ravidat, deviendront passeurs auprès du public, puisqu'ils seront parmi les premiers guides et surveillants de la grotte.

Les mécanismes de la légende sont désormais en marche. Les récits des découvreurs vont devoir s'y affronter et négocier avec elle. A Altamira comme à Lascaux, une figure centrale amène à la découverte des peintures. Le personnage de Maria trouve un alter ego dans la figure du chien de Marcel Ravidat. L'animal devient un motif central du récit, un symbole de la découverte et un élément omniprésent dans les dispositifs de médiation: l'arrière-train du chien en train de fouir le sol est sculpté à côté de la grotte originale, et ses aboiements retentissent sur le parcours qui conduit les visiteurs vers le fac-similé à Lascaux CIAP.

27 Cependant, au contraire de l'enfant, dont l'existence et le rôle semblent admis par tous avec plus ou moins d'enthousiasme, le chien de Lascaux semble être devenu la métaphore des débats sur l'authenticité des récits de découverte. Le premier article du Petit Parisien mentionne certes son existence, mais le chien disparaît dès le 27 septembre dans l'article de l'Echo du Centre et il n'est plus mentionné dans les articles suivants.

Cette authenticité des épisodes de la découverte est un point particulièrement sensible chez les découvreurs. Dans sa conférence donnée au musée de l'Homme en 1986, Jacques Marsal revient sur la présence du chien et le place dans un rapport particulier à la légende. Pour lui, comme la légende doit se nourrir de ponctuations anecdotiques, sans lesquelles le récit à destination du public ne pourrait se déployer pleinement, le chien doit être présent : "C'était entré dans la légende et, moi-même, je racontais aux touristes que c'était pour aller chercher le chien qu'on avait découvert la grotte " (Delluc B et Delluc $\mathrm{G}$ 2012, 552, note 2). Mais le langage de la vérité, qui échappe à la légende, et qui est celui des initiés, est autre : «Je vais vous parler de l'histoire du chien (...) Et quand c'est entré dans la légende, c'est difficile de l'en faire sortir. Je peux vous affirmer que le 
jour où on a découvert Lascaux, il n'y avait pas de chien » (Delluc B et Delluc G 2012, 305). Pourtant, de son côté, Marcel Ravidat livre un autre récit dans sa lettre de $1983^{12}$ : «mon chien bondi (sic) dans un fouré (sic) et ne ressortit plus ». La présence du chien n'est pas un point fondamental des débats sur Lascaux, loin s'en faut, mais son questionnement est significatif de l'incertitude de la fixation du récit, qui n'a jamais pu se constituer en histoire, mais reste légende, avec des faits dont la nature fluctue.

Si Lascaux est un mythe, c'est avant tout un mythe social, au sens de Georges Sorel ${ }^{13}$, le réceptacle d'un ensemble de représentations et de discours imbriqués, nés du choc de la découverte, qui fait émerger en vrac des images capables d'évoquer spontanément et simultanément de multiples constructions, qu'elles soient réelles ou non. A Lascaux, le régime de scientificité s'insère dans le mythe, il le nourrit, et le récit se fonde sur une imbrication entre construction scientifique, récit événementiel, et conte.

\section{Le récit avorté, ou la possibilité d'une grotte}

30 La grotte de Lascaux devient un modèle rayonnant et prégnant aussi bien dans les schémas de représentation que dans les espaces physiques. Elle entraine dans son sillage nombre d'autres grottes, qui seront désignées comme «le Lascaux de la sculpture $»^{14}$ (Roc-aux-Sorciers, découvert en 1950), "le Lascaux de Mayenne $»^{15}$ (grottes de Saulges, découvertes en 1967), "le Lascaux sous la mer $»^{16}$ (Cosquer, découverte en 1985), «le Lascaux de l'Age du Bronze $»^{17}$ (grotte des Fraux, découverte en 1989) ou «le Lascaux de la gravure $»^{18}$ (Cussac, découverte en 2000).

31 Si le rayonnement du mythe social s'étend à d'autres grottes éloignées de Montignac, à plus forte raison, l'espace physique du village qui a révélé Lascaux reste imprégné de la possibilité de l'avènement d'un autre épisode aussi intense que celui de 1940. Fin 2013, alors que le projet de Lascaux Centre international de l'art pariétal monte en puissance ${ }^{19}$, cette hypothèse d'une deuxième grotte originale s'affiche dans la presse, livrant un nouvel ensemble de représentations qui redéfinit le périmètre du mythe social de Lascaux.

Alors que l'on pouvait qualifier d'immédiate la temporalité des récits de la découverte de Lascaux, le récit de la possible découverte d'une nouvelle grotte ornée s'inscrit dans une temporalité indéfinie et suspendue, caractéristique d'une parousie. Cette temporalité particulière exclut les récits scientifiques, pour laisser le champ ouvert au récit journalistique, qui peut se déployer tant que l'objet n'est pas découvert. Un premier article parait dans Sud Ouest le 12 septembre, date anniversaire de la découverte de la grotte, signé par Adrien Vergnolle. Intitulé «A la recherche de la nouvelle Lascaux »", il présente précisément les éléments de cette quête. L'auteur insiste sur l'importance du mythe, qui engendre le désir : «Le mythe est né avec Lascaux (...) personne n'imagine Lascaux comme restant le seul témoignage aussi divin d'une humanité balbutiante (...) À l'époque [de la découverte], c'était amusant, tous les enfants sont devenus spéléologues ». Le désir n'est pas vain, dans la mesure où des découvertes exceptionnelles ont bien toujours lieu, et Adrien Vergnolle cite la découverte de la grotte de Cussac par un spéléologue en 2000. Ce désir est entretenu également par Laurent Mathieu, maire de Montignac, qui est lui aussi prêt à l'éventualité d'une découverte : «Ce n'est pas possible qu'il n'y ait que Lascaux (...). Il est tout à fait possible que ces hommes aient peint beaucoup plus, ailleurs. Ou tout près. ». 

deux forces extérieures au territoire: l'action de l'État et la fréquentation touristique. Liée à la réglementation archéologique, l'action de l'État engendre une crainte de dépossession: «Il arrive régulièrement qu'un témoignage annonce l'existence d'une grotte (...) qu'on aurait rebouché (sic) par crainte d'être exproprié. C'est assez crédible : chaque "propriétaire" d'une grotte ornée (celui qui possède le dessus possède le dessous) en a été dépossédé. Et en a tiré plus d'ennuis que de richesses. Quand l'État a commencé à sanctuariser la colline de Lascaux, les agriculteurs du coin ont frémi : on ne prendrait pas leurs terres, fussent-elles le manteau d'un mystère mondial ». Le désir de découverte est ainsi largement bridé par une inquiétude, qui vient teinter le mythe d'une autre couleur. Alors que la découverte de Lascaux constitue dans ses premiers temps un moment d'euphorie et d'enthousiasme unanime, la découverte potentielle d'une "nouvelle Lascaux » est d'emblée perçue comme une source de contentieux qui opposera les particuliers à l'État. L'image d'un propriétaire forcément « dépossédé » de son patrimoine renvoie à celle d'un Etat coercitif, ce qui s'oppose pourtant à la réalité de la gestion nationale des grottes ornées ${ }^{21}$. est un autre motif qui engendre le réflexe du secret. Pour l'occasion, c'est le scientifique qui s'exprime sous la plume du journaliste. Citant les paroles de Randall White, professeur d'anthropologie à l'université de New York, Adrien Vergnolle poursuit : «[Lascaux] a été conservée 18000 ans et en moins d'un siècle, on a réussi à l'abîmer. Malheureusement, l'une des plus belles grottes au monde sert de modèle de ce qu'il ne faut plus faire. (...) Trouver la nouvelle Lascaux ? "Au fond, il vaudrait mieux ne rien dire, alors." ». Cette deuxième inquiétude ne porte plus sur le moment ponctuel de la découverte mais se déploie dans le temps long de la conservation de la grotte. Les crises successives que la grotte de Lascaux a connues en matière de conservation sont autant de moments de crainte que l'on projette dans l'avenir. Le désir de revivre la magie de la découverte est bridé par la peur de reproduire les erreurs passées. La future grotte devra être capable de donner une joie identique à celle de Lascaux, puisqu'elle sera, dit le journaliste, «aussi belle » qu'elle, mais sa beauté même, dans les nouvelles configurations du mythe, est porteuse de destruction. C'est un prix que le découvreur n'est pas prêt à payer, il préfère donc garder le secret, c'est-à-dire interrompre le récit.

Un épisode de découverte avortée surgit trois mois plus tard à Montignac, aux environs de Noël. Celui-ci met en relief une nouvelle tension, qui emprunte autant au modèle de Lascaux qu'au modèle d'Altamira. Le récit de la découverte présente dans sa phase initiale de grandes analogies avec celui de Lascaux mais bifurque très vite pour s'opposer, comme à Altamira, à la validation scientifique, mais avec plus de force encore : à Altamira, c'était l'authenticité des peintures qui était remise en cause, dans ce nouvel épisode, c'est l'existence même de la grotte.

Dans le village, la rumeur court de l'existence d'une grotte qui aurait été découverte il y a longtemps sur le territoire de la commune de Montignac, près de Lascaux, cachée, puis perdue. Comme pour Lascaux, la temporalité des récits est rapide. Un sondage effectué sur la presse en ligne, permet de dénombrer quatre articles parus entre le 26 et le 31 décembre 2013 et un cinquième daté du 28 janvier 2014 : Sud-Ouest du 26 décembre ( Une autre Lascaux sous terre »), Libre Belgique du 31 décembre (« "Un nouveau Lascaux!”, la rumeur qui affole la Dordogne ») ${ }^{22}$, Midi Libre du 31 décembre ( Suspense en Dordogne : il existe peut-être une "autre" grotte de Lascaux »)23, France Bleu Périgord du 30 décembre 
(«Une deuxième grotte de Lascaux à Montignac: mythe ou réalité? ?) ${ }^{24}$, Journal du Dimanche du 28 janvier ("A la recherche du Lascaux oublié») ${ }^{25}$. Cette soudaine prolifération n'est pas uniquement de l'agitation journalistique, mais rend réellement compte de l'atmosphère enfiévrée qui a traversé Montignac cet hiver là, comme nous l'ont rapporté plusieurs témoignages ${ }^{26}$.

L'article paru dans Sud-Ouest du 26 décembre, contient l'essentiel des éléments qui sont repris dans les autres journaux. L'article se lit comme une réalisation, voire une épiphanie, des éléments exposés dans l'article précédent, également rédigé par Adrien Vergnolle. Une dame âgée rend visite au maire de Montignac, celui-là même qui avait évoqué en septembre la possibilité de l'existence d'une autre grotte. Elle lui rapporte qu'en 1962 deux frères, dont elle a épousé le cadet, ont vu à travers une cavité une peinture au fond d'une grotte, qu'ils referment aussitôt, non sans avoir récupéré des silex et une lampe à graisse du même type que celles découvertes à Lascaux. Interviewé par Adrien Vergnolle, le maire déclare : « Une chose est certaine : un homme a vu à travers un orifice l'arrière-train d'un animal, peint sur la paroi, à la façon de Lascaux ». Mais les deux frères choisissent de garder le secret et referment la cavité : «Donc, il y aurait quelque part dans la forêt une caverne peinte, peut-être aussi belle que l'autre ».

Ce récit emprunte beaucoup au récit de la découverte et à l'histoire de Lascaux, mais il se construit autant en référence qu'en opposition à eux. Dans les deux cas, c'est un trou ${ }^{27}$ qui s'ouvre, une figure qu'on aperçoit, une lampe et du mobilier. Mais le rebouchage du trou entraîne une interruption du récit, la perte du lieu de la découverte, et des œuvres qu'elle renferme. En ne rendant pas public leur découverte, les deux frères ont refusé de la confronter au régime de scientificité et l'ont plongée dans le secret. Mais, alors qu'à Lascaux les jeunes inventeurs ne gardent le secret que quelques jours, permettant de renouer le fil du récit, dans la "nouvelle Lascaux », le secret durera plus d'un demi-siècle, effaçant la mémoire mais multipliant les fils du récit.

Dans les deux cas, le processus de divulgation de la découverte emprunte des canaux analogues, mais leur nature a changé. Si en 1940, l'autorité incarnée par l'abbé Breuil projette instantanément le récit dans le régime de scientificité ; en 2013, l'autorité incarnée par la DRAC, administration déconcentrée de l'État, va construire le régime de scientificité contre le récit, comme à Altamira.

Le rapport entre l'autorité et le particulier constitue l'enjeu central du récit mais celui-ci est également instrumentalisé pour exprimer des préoccupations et des interrogations actuelles face à la gestion du patrimoine par l'État et à son imbrication avec les patrimoines familiaux. Les raisons invoquées par le journaliste pour expliquer la décision des deux frères semblent totalement anachroniques. Lascaux, exploitée par un propriétaire privé depuis 1948, « accueille 30000 visiteurs en 1955 et finalement 100000 en $1960 »$ (Geneste 2011, 56). Quant aux premières mesures de sanctuarisation de la colline, elles ne datent que de $2007^{28}$. En 1962, la découverte d'une grotte offre encore pour un particulier la perspective d'un revenu financier et non celle de tracasseries administratives. Si les frères ont eu à choisir de garder le secret, ce ne peut être que pour d'autres raisons, qui peuvent être très diverses.

41 Il n'en demeure pas moins que c'est bien l'État qui est au cœur du récit, en tant que garant du régime de scientificité et de la gestion du patrimoine. Dans son insistance à opposer l'intérêt des particuliers aux dispositions de l'État, l'article semble vouloir développer une métaphore de la perversion patrimoniale de l'État, dont l'action, au lieu de favoriser connaissance et diffusion, engendre secret et repli. Le fil du récit commencé 
avec la découverte de Lascaux est en passe d'être renoué, car le témoignage a fait resurgir l'histoire. Mais lorsque le récit essaye de redémarrer, la confrontation avec le régime de scientificité va l'empêcher de se dérouler.

Le recours au maire, intermédiaire entre le local et le national, fait entrer le récit dans une autre étape, celle de la validation par l'État. Le maire suit la procédure réglementaire de déclaration des découvertes archéologiques, en faisant appel aux services déconcentrés de l'archéologie. Le récit change alors de nature, il se déploie dans l'espace réglementaire et scientifique à la fois et le texte qui résume le résultat des recherches prend la forme d'un communiqué diffusé par la préfecture de la Dordogne ${ }^{29}$ :

«A la suite du signalement de la découverte ancienne (1962) d'une grotte ornée à Montignac par voie de presse (article journal Sud-Ouest du 26 décembre 2013), la vérification du potentiel archéologique du secteur concerné a été effectuée sous la direction du service régional de l'archéologie de la Drac Aquitaine.

Ces travaux de recherche ont été réalisés avec l'accord formel des propriétaires des terrains concernés.

La prospection pédestre réalisée, alliant observations géomorphologiques et archéologiques, a été complétée par une cartographie et un relevé à la caméra thermique.

La zone prospectée contient un ou plusieurs réseaux karstiques qui sont tous anciennement comblés, avec des décolmatages superficiels. Aucun indice d'une cavité présentant un vide un peu conséquent ni aucun indice de cavité obturée n'a été reconnu.

A partir de ce constat, il peut être affirmé qu'à ce jour, ce secteur ne révèle pas l'existence d'une grotte ornée et ne présente pas d'intérêt archéologique. »

Comme à Altamira, découverte et authentification s'opposent. Mais cette fois-ci, on ne fait pas appel à l'autorité en charge du régime de scientificité pour authentifier la découverte. On la charge bien au contraire de découvrir - ou de redécouvrir - un bien patrimonial à la fois familial et national donné d'emblée par le récit comme authentique. Mais au moment où le fil du récit tissé avec Altamira et Lascaux semble se renouer, l'institution qui s'est emparée, malgré elle, du récit, le fait avorter. La découverte ancienne non publicisée débouche alors, malgré la publication du récit, sur une nondécouverte.

Pourtant, si le régime de scientificité referme le récit, il se retrouve pris au piège. En s'appuyant sur le principe de réfutabilité qui est, selon Popper, un des fondements de la légitimité scientifique, et en déclarant qu' «à ce jour » l'existence d'une grotte n'est pas prouvée, le récit de l'État ouvre une brèche temporelle. La réponse du maire de Montignac ne se fait pas attendre: "“C'est sur le témoignage d'une habitante de Montignac que nous avions demandé ces investigations et je trouve étonnant qu'on ait engagé autant de moyens [...], sans écouter son témoignage". Il estime également que la dame avait nommé un lieu précis et que "ce n'est pas tout à fait là que les recherches ont été faites" ${ }^{30}$. Le mythe se nourrit non pas d'un, mais de plusieurs récits. Le récit ne pourra donc jamais mourir.

\section{Raconter et prouver}

La science préhistorique naissante a eu pour ambition de construire un régime de scientificité fondé sur une systématisation de la réalité observée, dans la logique de la preuve. Or, comme toute science humaine (Ricoeur 1983-1985), la discipline préhistorique a dû raconter afin de prouver. Mais les récits n'ont pas porté uniquement sur la 
démonstration de la rigoureuse construction de typologies, tant il a fallu constamment réajuster le régime de la preuve à celui de la découverte. Ils ont dû également rendre compte d'une découverte qui bouleversait le statut de l'homme préhistorique, puis, un demi-siècle plus tard, du jaillissement, au cœur de la guerre, d'un bestiaire rouge et noir et, plus tard encore, négocier avec l'émerveillement et la peur, qui imprégnera désormais toute possibilité ou tout désir de nouvelle découverte. Comment prouver ce qui est source de désordre, engendrant, à Altamira, de profondes remises en question scientifiques, et à Lascaux, un débordement de surqualifications esthétiques?

La narration d'un récit d'observation, comme Sautuola a tenté de le faire, devait se révéler inefficace. C'est la légende qui sera seule capable d'égaler le saut considérable que le régime de scientificité doit accomplir pour inclure l'art pariétal dans les paradigmes de la science préhistorique du XIX siècle. Alors que l'espace de communication envers un large public se déploie sans cesse davantage, l'espace des récits scientifiques ne s'agrandit pas. Cette cohabitation caractérisée par une déséquilibre croissant engendre un régime de reconnaissance particulier. La légende, née de ce régime, va grandir et dialoguer avec lui. A Lascaux, face à l'exceptionnalité de l'œuvre, couplée au contexte non moins exceptionnel de la découverte, seul le mythe peut être à la hauteur du régime de scientificité. Mais parce qu'il s'est adossé au merveilleux et au sacré, le mythe se construira en un système logique et imparable qui s'affranchit de toute validation. Lorsque surgit la possibilité d'une grotte, le récit issu du mythe fait fi du régime de scientificité. L'interruption d'un récit n'entraine plus sa disparition.

L'impossibilité de cette disparition interroge le lien entre récit et preuve. Dans les récits de découverte ou de non-découverte des grottes ornées, la tension entre nécessité de la preuve et récit naît de la discordance entre la logique d'empilement de la construction de la preuve scientifique et la temporalité du récit : un événement, de quelque nature qu'il soit, se produit dans un contexte donné à un moment donné, alors que ce sont plusieurs récits accumulés qui composent le régime de scientificité. Or, dans les découvertes de grottes ornées, le récit, tel qu'il est né avec Altamira pour se fixer sur le modèle de Lascaux, a fini par adopter une logique au sein de laquelle plusieurs départs peuvent être amorcés à n'importe quel moment, engendrant ainsi une structuration buissonnante des récits. Dans certains cas, comme avec Lascaux, ces départs peuvent coïncider avec la logique de la science, dans d'autres, comme avec la grotte perdue de Montignac, ils peuvent continuer leur existence sans nécessité de la preuve. Le récit est devenu le produit du régime de scientificité, mais il est également devenu une preuve dans la logique mythique qui s'est mise en place. Pour emporter la conviction, raconter équivaut à prouver.

\section{BIBLIOGRAPHY}

Anonyme, 1884. « Visite aux cavernes de Santillana, près Santander ». In Congrès international d'Anthropologie et d'Archéologie préhistoriques, $9^{e}$ session, Lisbonne, 1880, 47. Lisbonne : Typographie de l'Académie royale des sciences. 
Boudreau, C. et Arseneault, A. 1994. «La recherche qualitative : une méthode différente, des critères de scientificité adaptés ». Recherche Qualitative 10 :121-137.

Bethlenfalvay, M. 1979. Les Visages de l'enfant dans la littérature française du XIX ${ }^{e}$ siècle. Genève : Droz.

Bras, G. 2006. « L'Homme qui tua Liberty Valance, ou la constitution imaginaire du peuple ». Rue Descartes, 53 (3) : 31-45.

Cartailhac, E. 1902. « Les Cavernes ornées de dessins, la grotte d'Altamira, Espagne, "Mea culpa" d'un sceptique ». L'Anthropologie 13 : 348-354.

Cartailhac, E. et Breuil, H. 1906. La caverne d'Altamira à Santillane près Santander (Espagne), Monaco : Imprimerie de Monaco.

Coye, N. 1997. La préhistoire en parole et en acte. Méthodes et enjeux de la pratique archéologique (1830-1950). Paris : l'Harmattan.

Coye, N., 2000. « En leur science et conscience. Vulgarisateurs et caution scientifique en préhistoire au XIX siècle ». In L'Homme préhistorique. Image et imaginaire, Ducros A. et J. ed. Paris : l'Harmattan.

Déchelette, J. 1908. Manuel d'archéologie préhistorique celtique et gallo-romaine, t. I: Archéologie préhistorique. Paris : Alphonse Picard et fils éditeurs.

Delluc B. et Delluc G. 2003. Lascaux retrouvé. Périgueux : Pilote 2.4 édition

Delluc B. et Delluc G. 2012. « Lascaux et la presse des années 1940 ». Bulletin de la Société Historique et Archéologique du Périgord 139 : 551-577.

Delluc B., Delluc G et Strauel J.P. 2016. «La découverte de Lascaux en 1940. Du nouveau avec les Alsaciens ", Bulletin de la Société Historique et Archéologique du Périgord 143 : 361-374.

Drapeau, M. 2004. « Les critères de scientificité en recherche qualitative ». Pratiques psychologiques : 10, 79-86

Fabre, D. 2008. « Le poète dans la caverne. Georges Bataille à Lascaux ». In Imaginaires archéologiques, Voisenat C. ed., Paris : éditions de la Maison des Sciences de l'Homme, p. 127-182

Félix, T. et Bigotto P. 1990. Le Secret des bois de Lascaux. s.l. : Impact (Zigzag)

Félix, T. et Bigotto P. 2008. Le Secret des bois de Lascaux. Sarlat : Dolmen

Fraenkel, B. 2010. «L'invention de l'art pariétal préhistorique », Gradhiva [En ligne], 6 | 2007, mis en ligne le 15 novembre 2010, consulté le 5 août 2017. URL : http://gradhiva.revues.org/984

Gaussel, A. 1997. Le Récit de découverte des grottes ornées paléolithiques. Mémoire de DEA : Préhistoire et anthropologie. Aix-en-Provence : Université de Provence - UFR Civilisations et Humanités.

Geneste, G. 2011. « les grandes étapes de la conservation de Lascaux ». In Lascaux et la conservation en milieu souterrain, Actes du symposium international, Paris, 26 et 27 février 2009, Coye N. éd. Paris : éditions de la Maison des Sciences de l'Homme (Documents d'archéologie française) : 51-72.

Harlé, E. 1881. « La grotte d'Altamira près de Santander (Espagne) », Matériaux pour l'histoire primitive et naturelle de l'homme 16 : 275-283.

Hurel, A. 2007. La France préhistorienne de 1789 à 1941. Paris : CNRS Editions.

Kahn, P. 1951. « Mythe et réalité sociale chez Sorel ». Cahiers Internationaux de Sociologie 11:

131-154.

Larivaille, P. 1974. «L’analyse (morpho)logique du récit », Poétique 19 :368-388. 
Lasheras J.A. et las Heras C. éd. 2004. «Introduction », dans Sautuola, M. Sanz de. Breves apuntes sobre algunos objetos prehistóricos de la provincia de Santander. Traduction française. Madrid:

Turner.

Lecompte, M.D. et Goetz, J. 1982. « Problems of reliability and validity in ethnographic research ». Review of Educational Research $52: 31-60$.

Pernoud E. 2003. L'invention du dessin d'enfant en France, à l'aube des avant-gardes. Paris : Hazan.

Richard, N., 1993. «De l'art ludique à l'art magique ; Interprétations de l'art pariétal au XIX ${ }^{\mathrm{e}}$ siècle ». Bulletin de la Société préhistorique française 90 (1), 60-68.

Richard, N., 2008. Inventer la préhistoire-Les débuts de l'archéologie préhistorique en France. Paris : Vuibert/Adapt-SNES.

Ricoeur, P. 1983-1985. Temps et histoire. 3 vol. Paris : Seuil.

Rivière, E. 1902. « Excursion de la section aux Eyzies ». In Congrès de l'Association française pour l'avancement des sciences, $31^{e}$ session, Montauban, 1902, 1 : 271-272. Paris, Secrétariat de l'association.

Sautuola, M. Sanz de 1880. Breves apuntes sobre algunos objetos prehistóricos de la provincia de Santander. Madrid : Murillo.

Sorel, G., 1908. Réflexions sur la violence. Paris : Marcel Rivière et C ${ }^{\text {ie }}$.

Vilanova y Piera, J. 1883. «Sur la caverne de Santillana », In Congrès de l'Association française pour l'avancement des sciences, $11^{e}$ session, La Rochelle, 669-693. Paris : Secrétariat de l'Association.

\section{NOTES}

1. Une vision synthétique de ce débat est disponible en ligne sur le site du Pôle International de la Préhistoire: http://pole-prehistoire.com/index.php/es/74-francais/fr-decouvrir/frexpositions-virtuelles/histoire-de-la-prehistoire/156-la-reconnaissance-de-l-art-parietal? showall=\&start $=1$

2. Archéologue de terrain, divulgateur et animateur de la recherche préhistorique dans les Pyrénées avec un prolongement de ses recherches dans la péninsule ibérique à partir de 1881 - Emile Cartailhac appartient à la génération qui assure la transition entre les fondateurs de la science préhistorique et les chercheurs qui refondent la discipline dans les premières décennies du XXe siècle.

3. Les auteurs consacrent une longue introduction au débat sur la reconnaissance d'Altamira. Ils mentionnent le fait que c'est après en avoir été «averti par sa jeune fille » que Sautuola s'est intéressé aux peintures (Cartailhac et Breuil 1906, 2, 12 et 68)

4. Autobiographie, chapitre XII, folio 183, Musée d'Archéologie Nationale, fonds Breuil

5. «Le "héros" est alors celui qui ajuste, au mieux de la cohésion de la communauté, l'ensemble de ses parties. » (Bras 2006, 45).

6. Les quatre découvreurs « officiels » de la grotte sont Jacques Marsal, Simon Coencas, Georges Agniel et Marcel Ravidat. Depuis ces dernières années, les recherches de Brigitte et Gilles Delluc ont montré que ces derniers ont été accompagnés d'au moins trois autres, deux adolescents et une adolescente. Voir Delluc B. et G, Strauel J.P., 2016.

7. Voir le témoignage de Jacques Marsal lors de sa conférence donnée au musée de l'Homme le 14 octobre 1986: «Il y avait quand même cette petite idée de souterrain, de trésor (...). Et ce que nous cherchions, nous, ce n'était pas tellement les grottes préhistoriques. On allait dans les trous, sous les abris, on jouait (...). On est arrivé à Lascaux avec cette idée de trésor.» (cité dans Delluc B. et Delluc G. 2003, 303). 
8. L'Echo du Centre, 28 septembre 1940

9. http://www.dailymotion.com/video/xedbn_lascaux_shortfilms

10. Témoignage de Léon Laval, d'après des notes extraites d'un «petit livret rédigé par l'instituteur » en 1949 (Delluc B et Delluc G. 2003, 308).

11. L'abbé Breuil emploie dans cette expression dans une conférence qu'il donne à Montignac le 17 décembre 1940 (Delluc B. et Delluc G. 2012). Elle est en fait directement inspirée d'une expression employée par Joseph Déchelette qui avait qualifié Altamira de «Chapelle Sixtine de l'art quaternaire » (Déchelette 1908, 150).

12. Cette lettre qui figure dans le dossier documentaire de l'édition de 1990 du Secret des bois de Lascaux, sera supprimée des éditions successives (voir par exemple Félix et Bigotto 2008).

13. Voir en particulier ses Réflexions sur la violence (Sorel 1908) et l'étude de Paul Kahn (Kahn 1951).

14.

http://www.lexpress.fr/actualite/sciences/le-roc-aux-sorciers-le-lascaux-de-lasculpture_1641367.html

15. http://www.ouest-france.fr/culture/les-grottes-de-saulges-le-lascaux-de-mayenne-5126773

16. http://www.lexpress.fr/informations/un-lascaux-sous-la-mer_597293.html

17. Sud-Ouest, 15 décembre 1989

18. http://www.ladepeche.fr/article/2001/07/05/219742-la-grotte-de-cussac-est-un-lascaux-dela-gravure.html

19. Le projet de Lascaux CIAP est présenté publiquement en décembre 2012, la première pierre est posée en avril 2014 et le chantier est lancé au mois de juin suivant.

20.

http://www.sudouest.fr/2013/09/12/a-la-recherche-de-la-nouvelle-

lascaux-1166500-2224.php

21. En effet, sur les 178 grottes et abris ornés paléolithiques comptabilisés sur le territoire métropolitain par le Centre National de Préhistoire, seulement $34 \%$ sont dans le domaine public, qu'ils appartiennent à l'État ou aux collectivités territoriales (chiffres mentionnés lors d'une conférence donnée par Geneviève Pinçon et Stéphane Konik au Pôle International de la Préhistoire le 16 juin 2017. Disponible sur http://pole-prehistoire.com/index.php/fr/decouvrir/ les-ressources-en-ligne/voir-ecouter)

22. http://www.lalibre.be/lifestyle/magazine/un-nouveau-lascaux-la-rumeur-qui-affole-ladordogne-52c2a62f3570105ef7e10219 - media

23. http://www.midilibre.fr/2013/12/30/suspens-en-dordogne-il-existe-peut-etre-une-autregrotte-de-lascaux,802814.php

24. https://www.francebleu.fr/infos/sante-sciences/une-deuxieme-grotte-de-lascauxmontignac-mythe-ou-realite- 1388382600

25. http://www.lejdd.fr/Societe/A-la-recherche-du-Lascaux-oublie-650587

26. Nous n'avons pas effectué de véritables entrevues sur le sujet, mais à chaque fois que nous avons mentionné cet épisode à des habitants de Montignac, des Eyzies-de-Tayac, de Tursac ou de Saint-Geniès, bourgades proches de Montignac, nous avons eu une réaction immédiate, qui ne faisait pas référence à des articles de presse, mais bien à une rumeur qui avait occupé la région pendant les fêtes de Noël.

27. A noter que «trou » dans le Périgord est synonyme de grotte, sans connotation péjorative.

28. La grotte de Lascaux Montignac - Dordogne - Nouvelle Aquitaine - France. Inauguration du centre international de l'art pariétal. 10 décembre 2016. Dossier de presse. www.culturecommunication.gouv.fr/content/.../1/.../20161209_MCC-DP-Lascaux.pdf

29. http://www.dordogne.gouv.fr/content/download/18322/141855/file/CP-résultats\% 20prospection\%20Montignac...pdf

30. "La deuxième grotte de Lascaux de Montignac n'existe pas », France TV Info, 14 février 2014 http://www.francetvinfo.fr/societe/la-deuxieme-grotte-de-lascaux-de-montignac-n-existepas_1681685.html 


\section{ABSTRACTS}

Starting in 1879, with the discovery of the paintings of the Altamira cave in Spain, the debate over the antiquity and authenticity of cave art developed in Europe for more than twenty years. The length of this debate reveals the difficulty for prehistorians of the late 19th century to integrate cave art into the scientificity regime, as defined by prehistorian practice since the late 1850s. The story of the discovery was quickly summoned to form the elements of a demonstration of another order, establishing a legend that continued to develop, even when the authenticity debate was closed.

Faced with this model in which the legend stems from the scientificity regime, the numerous stories of the discovery of Lascaux, Montignac (Dordogne), in 1940, reveal a different configuration. At the moment when the cave was immediately inserted, and without questioning, in prehistoric knowledge, the stories of the discovery had to deal with stories of authentication and analysis of the parietal device, making the Lascaux cave reach the rank of a social myth. With Lascaux, the story is based on an interweaving between scientific construction, event narrative, and storytelling.

The two archetypes of Altamira and Lascaux's cave discovery narrative weave a grid of analysis that enable us to address the development and treatment of a rumor that spread at the end of 2013 in the region of Montignac, about a "new Lascaux ", said to have been seen in the early 1960 s but never revealed and then lost. The process is reversed here $:$ it is no longer a question of telling the story of an unexpected discovery, but of bringing about the possibility contained in the story. The search resulted in a non-discovery, but this lack of scientific validation was not unanimously considered as a demonstration that could cut the story short. The story continues to develop, in a process where telling is equivalent to proving.

Le débat sur l'ancienneté et l'authenticité de l'art pariétal se développe en Europe pendant plus de vingt ans, à partir de 1879 où se place la découverte des peintures de la grotte d'Altamira en Espagne. La durée de ce débat révèle la difficulté pour les préhistoriens de la fin du XIXe siècle d'intégrer l'art pariétal dans le régime de scientificité, tel que la pratique préhistorienne l'a défini depuis la fin des années 1850. Mais le récit des conditions de la découverte va rapidement être convoqué pour constituer les éléments d'une démonstration d'un autre ordre, instituant une légende qui continuera à se développer, une fois le débat tranché.

Face à ce modèle dans lequel la légende naît du régime de scientificité, les nombreux récits de la découverte de Lascaux, à Montignac (Dordogne), intervenue en 1940, révèlent une configuration différente. Au moment où la grotte s'insère aussitôt et sans questionnement dans la connaissance, les récits de découverte composent avec les récits d'authentification et d'analyse du dispositif pariétal, faisant accéder la grotte de Lascaux au rang de mythe social. A Lascaux, le récit se fonde sur une imbrication entre construction scientifique, récit événementiel, et conte. Les deux archétypes de récit de découverte de grottes ornées d'Altamira et Lascaux tissent une grille d'analyse pour aborder le développement et le traitement de la rumeur qui se répand fin 2013 dans la région de Montignac, à propos d'une "nouvelle Lascaux », aperçue au début des années 1960 mais non révélée et aujourd'hui perdue. Le processus est ici inversé : il ne s'agit plus de faire le récit d'une découverte advenue, mais de faire advenir la possibilité contenue dans le récit. La recherche se soldera par une non-découverte, mais cette absence de validation 
scientifique ne sera pas unanimement considérée comme une démonstration pouvant couper court au récit. Celui-ci continue à se développer, dans un processus où raconter équivaut à prouver.

\section{INDEX}

Geographical index: aire franco-cantabrique, France, Espagne Chronological index: XIXe siècle, XXe siècle, XXIe siècle

Mots-clés: Altamira, Cantabrie (Espagne), Lascaux, Montignac (Dordogne), grottes ornées, art pariétal, préhistoire, récit de découverte, régime de scientificité, mythe, conte

\section{AUTHORS}

\section{SYLVIE GRENET}

Ministère de la Culture, direction générale des patrimoines IDEMEC - UMR 7307, Université d'Aix-Marseille sylvie_grenet@yahoo.fr

NOËL COYE

Pôle International de la Préhistoire

TRACES - UMR 5608 Université Toulouse Jean Jaurès noel.coye@pole-prehistoire.com 